\title{
MI CAL2 is a novel human cancer gene controlling mesenchymal to epithelial transition involved in cancer growth and invasion
}

\author{
Sara Mariotti ${ }^{1, *}$, I vana Barravecchia ${ }^{1, *}$, Carla Vindigni², Angela Pucci, Michele \\ Balsamoㄹ, Rosaliana Libro", Vera Senchenko5, Alexey Dmitriev ${ }^{5}$, Emanuela \\ Jacchetti ${ }^{6}$, Marco Cecchini ${ }^{6}$, Franco Roviello ${ }^{7}$, Michele Lai ${ }^{1,8}$, Vania Broccoli ${ }^{9}$, \\ Massimiliano Andreazzoli ${ }^{10}$, Chiara M. Mazzanti ${ }^{8}$, Debora Angeloni ${ }^{1}$

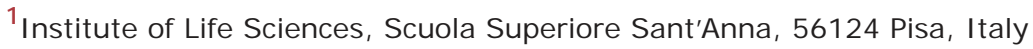 \\ ${ }^{2}$ U.O.C. Anatomia Patologica, Azienda Ospedaliera Universitaria Senese, Policlinico Le Scotte, 53100 Siena, Italy \\ ${ }^{3}$ U.O.C. Anatomia Patologica, Azienda Ospedaliera Universitaria Pisana, 56100 Pisa, Italy \\ ${ }^{4}$ BIOS Doctoral School in Life Sciences, University of Pisa, 56124 Pisa, Italy \\ ${ }^{5}$ Engelhardt Institute of Molecular Biology, Russian Academy of Sciences, 119991 Moscow, Russia \\ ${ }^{6}$ NEST, National Enterprise for nanoScience and nanoTechnology, CNR and Scuola Normale Superiore, 56127 Pisa, Italy \\ ${ }^{7}$ Department of Human Pathology and Oncology, University of Siena, 53100 Siena, Italy \\ ${ }^{8}$ Pisa Science Foundation, 56100 Pisa, I taly \\ ${ }^{9}$ DIBIT H San Raffaele, 20132 Milan, I taly \\ ${ }^{10}$ Department of Biology, University of Pisa, 56127 Pisa, Italy \\ *These authors contributed equally to this work
}

Correspondence to: Debora Angeloni, e-mail: angeloni@sssup.it and angeloni@ifc.cnr.it

Keywords: MICAL2, kidney cancer, gastric cancer, epythelial to mesenchymal transition, metastasis

Received: April 24, $2015 \quad$ Accepted: November 14, 2015

Published: December 12, 2015

\section{ABSTRACT}

The MI CAL (Molecules I nteracting with CasL) proteins catalyze actin oxidationreduction reactions destabilizing $\mathrm{F}$-actin in cytoskeletal dynamics.

Here we show for the first time that MI CAL2 mRNA is significantly over-expressed in aggressive, poorly differentiated/ undifferentiated, primary human epithelial cancers (gastric and renal). I mmunohistochemistry showed MI CAL2-positive cells on the cancer invasive front and in metastasizing cancer cells inside emboli, but not at sites of metastasis, suggesting MI CAL2 expression was 'on' in a subpopulation of primary cancer cells seemingly detaching from the tissue of origin, enter emboli and travel to distant sites, and was turned 'off' upon homing at metastatic sites.

In vitro, MICAL2 knock-down resulted in mesenchymal to epithelial transition, reduction of viability, and loss of motility and invasion properties of human cancer cells. Moreover, expression of MICAL2 CDNA in MICAL2-depleted cells induced epithelial to mesenchymal transition.

Altogether our data indicate that MI CAL2 over-expression is associated with cancer progression and metastatic disease. MI CAL2 might be an important regulator of epithelial to mesenchymal transition and therefore a promising target for anti-metastatic therapy. 


\section{INTRODUCTION}

The cure of most cancers will ultimately depend on the capability to eradicate disseminated secondary tumors that do not respond to therapy. Our current inability to do so motivates the search for target genes implicated in cancer progression.

Cytoskeleton remodeling is a crucial event in metastasis progression because it is instrumental in regulating fundamental cell properties, from proliferation and/or differentiation to cell-cell and cell-substrate adhesion, motility and invasion. Tight regulation of actin dynamics relies on the activity of different actin-binding proteins controlling the transition from globular (G) to filamentous (F) actin, as well as nucleation, capping, severing, elongation and crosslinking of actin filaments $[1,2]$.

Recently, the family of Molecules Interacting with CasL (MICAL) [3, 4], was implicated in the regulation of actin cytoskeleton dynamics [5, 6]. MICALs have a unique structure that combines an $\mathrm{N}$-terminal flavoprotein mono-oxygenase (MO) domain [7, 8] with other protein-protein interaction modules that network with cytoskeletal and signaling partners [3, 4]. Drosophila MICAL (D-MICAL) exerts oxidation-reduction (Redox) reactions to directly oxidize two methionine residues of actin, thereby destabilizing F-actin and inhibiting local assembly. D-MICAL activity is necessary for spatial guidance of the axonal growth cone, a highly motile sensory structure localized at the axon tip, essential for guiding neurons to their synaptic targets [9]. We reasoned that the striking capability of MICAL to directly and mechanistically connect oxygen availability with F-actin depolimerization and hence cytoskeleton dynamics might be extremely important also for metastatic cancer cells whose motility is increased as part of epithelial to mesenchymal transition (EMT). In fact, during the growth of solid tumors challenging micro-environmental factors (hypoxia, acidity, inflammatory cytokines, etc) stimulate cancer cells to enact escape adaptive strategies. Lead by a regulated genetic/epigenetic program, epithelial cells loose epithelial markers, cell-cell and cell-extracellular matrix (ECM) interactions, undergo cytoskeleton reorganization, gain gene expression profile, morphological and functional characteristics of mesenchymal cells, and leave the primary tumor site [10]. Both EMT and its opposite, mesenchymal to epithelial transition (MET), are implicated in developmental and pathological contexts [10]. During MET, mesenchymal markers are downregulated, cell motility decreases and cells adopt epithelial characteristics [10].

Up to now, MICALs involvement in human cancer was completely unexplored except for a report of MICAL2 splicing variants identified in prostate cancer [11]. While this work was in submission, it was published that MICAL-LIKE2, a protein of the MICAL family that shares sequence homology with MICAL2 but lacks the aminoterminal mono-oxygenase domain, is overexpressed in ovarian cancer and when silenced induces MET in ovarian cancer cells [12].

Given the relevance of MICAL proteins to cell motility and the complete lack of information in the context of human primary cancer, we were compelled to understand whether MICAL2 activity might affect cancer cell motility and/or invasion activity, two properties crucial for determining the magnitude of cancer clinical effect. So we set out to investigate a possible involvement of MICALs in human epithelial cancer. We started with MICAL2 because of its basal activation not downregulated by self-inhibitory activity present in MICAL1 and possibly in MICAL3 [13-15]. This feature suggested that deregulated expression might be sufficient to derange MICAL2 function, a trait in common to other actinbinding proteins involved in cancer.

\section{RESULTS}

\section{MICAL2 is variably expressed in human normal and cancer tissues}

To search for novel genes involved in metastasis, we investigated the possible role of MICAL2 in cancer.

Interrogating web-based, expression databases we found MICAL2 mRNA variably and almost ubiquitously expressed in normal tissues, including stomach, lung and kidney (UniGene: http://www.ncbi.nlm.nih.gov/ UniGene/ESTProfileViewer.cgi?uglist = Hs.501928), with noticeable expression variations in several types of human cancer (IST Online: http://ist.medisapiens. com/\#ENSG00000133816), together with several outliers, also in lung and gastric cancer (GC), indicating possible patient subpopulations within each cancer type. In vitro, comparing MICAL2 mRNA z-score across the NCI-60 panel, we found MICAL2 variably expressed in different cell lines (CellMiner: http://discover.nci.nih.gov/cellminer/ analysis.do). Interestingly, MICAL2 was down-regulated in epithelial-like breast cancer cells MCF7 and T47D, but up-regulated in breast cancer cells with mesenchymal features MDA-MB 231, BT-549 and HS578T.

We further explored the clinical significance of $M I C A L 2$ in human cancer performing comparative real time PCR (QRT-PCR) in normal/tumor paired biopsies of patients affected with three major types of human cancers: of the lung (non small cell lung cancer, NSCLC: Adenocarcinoma, AC; squamous cell carcinoma, SCC), kidney (clear cell renal cell carcinoma, ccRCC; papillary renal cell carcinoma, pRCC), and stomach (diffuse and intestinal histotypes).

In 27 NSCLC patients (11 AC, 16 SCC), we found statistically significant under-expression of MICAL2 in SCC primary tumors compared with AC $(p<0.01$, Figure 1A). We wondered whether it could be associated 
with SCC lesser tendency to be invasive compared with $\mathrm{AC}$, but this was not investigated further.

MICAL2 was moderately to highly over-expressed in $6 / 8$ pRCC patients (75\%, Figure 1A). In 36 ccRCC primary samples, we found MICAL2 significantly overexpressed in patients 'with metastases' versus 'without metastases' ( $p<0.05$, Figure 1B).

Three histotypes of GC are described [16]: 1) intestinal, (cancer cells are slow-growing, well differentiated, forming tubular or papillary gland structures), 2) diffuse (cancer cells are more aggressive, poorly differentiated, with a tendency to spread); 3) mixed (with growing characteristics common to both diffuse and intestinal histotypes). In 30 GC samples, MICAL2 was found over-expressed in the diffuse histotype compared with intestinal and mixed histotypes together ( $p<0.01$, Figure 1C). Further, MICAL2 was found overexpressed $(p<0.05)$ in samples of poorly differentiated and undifferentiated subtype G3 and G4 grade score (WHO criteria, [17]) as opposed to well and moderately differentiated G1 and G2 score [17], not shown.

Overall, QRT-PCR showed MICAL2 up-regulation in poorly differentiated, aggressive tumor of the lung, kidney and stomach compared with paired normal tissues, and in association with metastasis.

\section{MICAL2 is expressed at the cancer invasive front, and inversely correlates with expression of proliferation marker Ki-67}

To identify the cellular distribution of MICAL2 expression, we performed immunohistochemistry (IHC) analyses of histological sections of gastric and kidney cancer, using specific anti-MICAL2 polyclonal antibodies generated in our laboratory (Supplementary Figure 1).

In GC, IHC showed a patchy distribution of MICAL2 over-expression in comparison with normal surrounding mucosa in 31/40 samples (77.5\%). In diffuse, signet ring adenocarcinomas, MICAL2 expression was high within the tumor mass and in scattered neoplastic cells infiltrating the gastric wall (Figure 2A). Conversely, MICAL2 expression was undetectable in cancer cells of well differentiated, intestinal-type tumors (Figure 2B). MICAL2-positive cells were localized at the tumor infiltrating front (17/17 samples, 100\%, Figure 2C left) rather than within the neoplastic core $(p=0.0001$, Figure $2 \mathrm{C}$ right). Moreover, MICAL2-positive cells were found in neoplastic emboli (8/10 samples, $80 \%$, Figure 2D left), where MICAL2 expression was higher than in the neoplastic core ( $p=0.0038$, Figure $2 \mathrm{D}$ right), and where sometimes it showed both nuclear and cytoplasmic localization. When variable differentiation grades were present within the same tumor, MICAL2 immunolabelling was higher in the less differentiated areas. Endothelial cells (ECs) of neo-angiogenic capillaries that extensively branch into the cancer mass showed a very intense MICAL2 immunostaining (Figure 2B), but no expression of MICAL1 and MICAL3 (Supplementary Figure 2), suggesting a different role of MICAL proteins within neoangiogenic ECs.

Given the peculiar localization of MICAL2-positive cells on the cancer invasive front, we wondered how this would correlate with the tumor cells proliferative state. Therefore, we analyzed the expression of Ki-67, a ubiquitous cell proliferation marker [18]. Consecutive sections from the same paraffin blocks were used for either immunostaining of MICAL2 or Ki-67. We found Ki-67 expression level inversely correlated with MICAL2 expression ( $p<0.05$, Figure $2 \mathrm{E}$ ).

In ccRCC, the subcellular localization of MICAL2 was either cytoplasmic (Figure 3A), nuclear (Figure 3B), or both (Figure 3C) with cellular pattern variability within the same tumor. In addition, MICAL2 was highly expressed in ECs of the numerous cancer-associated capillaries (Figure 3D). Noticeably, MICAL2-positive neoplastic cells were detected within tumor emboli of lung metastases (Figure 3E) whereas, in the remaining cells of metastatic lesions to the brain (not shown) and lung (Figure 3F), MICAL2 protein expression was very low, localized to rare and scattered peripheral cells within the metastatic mass. MICAL2 protein was low in normal tubules and undetectable in normal glomeruli (Supplementary Figure 3).

Although it was not feasible to evaluate systematically and quantitatively the cancer invasive edge in the ccRCC samples available for this study, qualitative IHC analysis of single-antigen immunolabelling showed Ki-67 (Figure 3G) to be mainly expressed in the tumor core where MICAL2 (Figure $3 \mathrm{H}$ ) was not or scarcely expressed, and vice versa (Figure 3I, 3J). So, MICAL2 expression inversely correlated with proliferation also in kidney cancer.

Altogether, data showed MICAL2 expression was high in aggressive primary cancer and in metastatic emboli, but barely or not detectable in cancer cells at metastatic sites.

\section{Depletion of MICAL2 in cancer cells induces MET in vitro}

With knock-down experiments, we investigated MICAL2 loss-of-function in human cancer cell lines with mesenchymal phenotype derived from kidney (786-O), breast (MDA-MB-231) and pleural cavity (MERO-14) respectively (generated as in Supplementary Figure 4). We performed RNA interference with commercial siRNAexpressing plasmids, using three specific siRNAs for the target of interest, plus one non-specific siRNA and the corresponding empty vector as references. All through the text, cell lines are designated as follows: parental, untreated cancer cell lines as 'WT'; cells transfected with control plasmid (either TRCN0000192634 or empty pLKO.1-Neo-CMV-tGFP) as 'CTRL' cells; 786O MIC2-KD clones as KD2 (obtained from tranfection 
with a single plasmid, TRCN0000046581) and KD14 (obtained from simultaneus transfection with three plasmids plasmids TRCN0000046579, TRCN0000046580 e TRCN0000046581); MERO-14 MIC2-KD cells as KDM1; MDA-MB-231 MIC2-KD cells as KDM4. (KDM1 and KDM4 were obtained from transfection with all three plasmids). CTRL and parental cells are collectivey referred to as 'reference' cells. For details, see Materials and Methods and Supplementary Materials.

786-O MIC2-KD cells immediately appeared morphologically different (Figure 4), regardless of cell density in the culture plate (not shown). Instead of
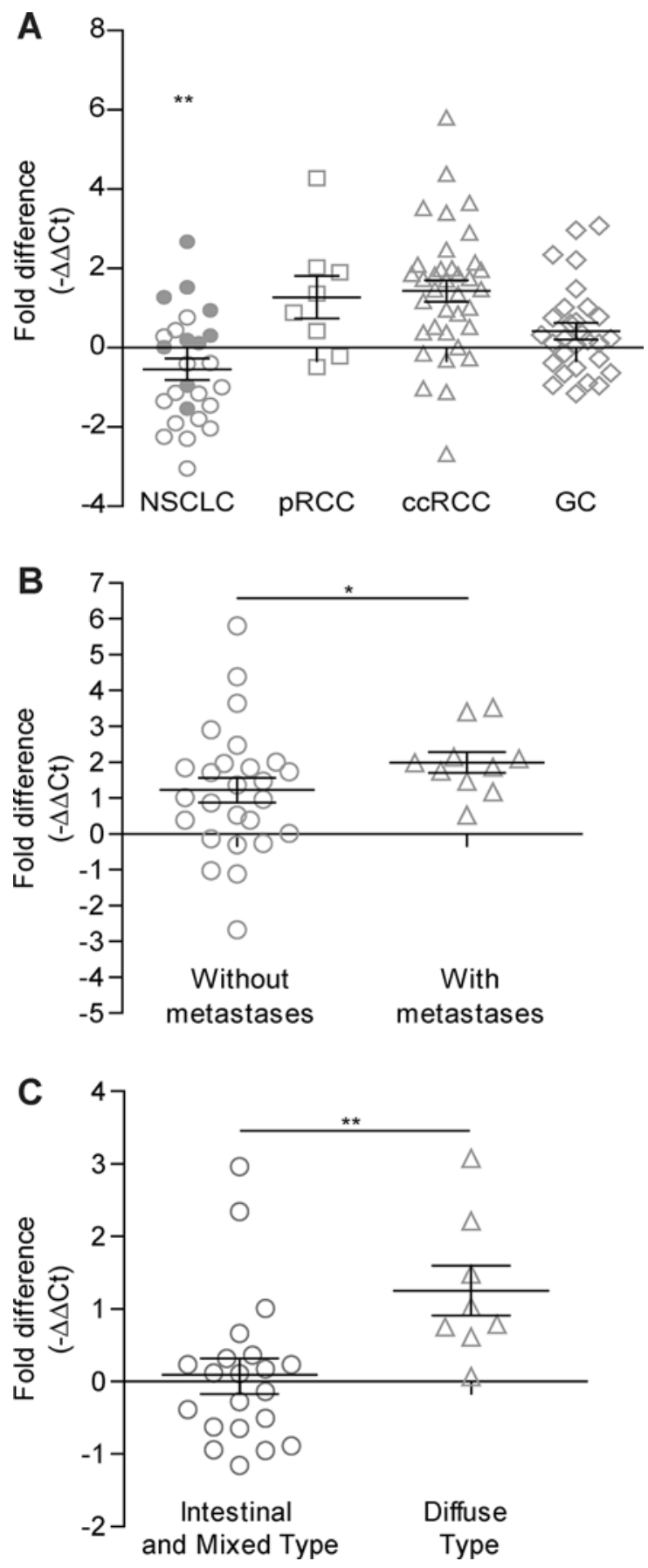

Figure 1: QRT-PCR gene expression analysis of MICAL2 in lung, kidney and stomach cancers. (A) MICAL2 deregulated expression in NSCLC (circles, $N=26$ ), pRCC (squares, $N=8$ ), ccRCC (triangles, $N=36$ ) and GC (diamonds, $N=29$ ). In NSCLC patients (10 AC and 16 SCC), MICAL2 is under-expressed in SCC (open circles, $N=16$ ) in comparison to AC (thick circles, $N=10$ ) patients. (B) MICAL2 mRNA level in ccRCC $(N=36)$ samples, against normal mucosa, was higher in patients with metastasis (triangles) in contrast to those without (circles). (C) In GC ( $N=29), M I C A L 2$ was over-expressed in Diffuse type (triangles) versus Intestinal or Mixed type (circles) samples. In all graphs, horizontal lines denote mean and SEM. ${ }^{*} p \leq 0.05$, ${ }^{* *} p \leq 0.01$, ${ }^{* * *} p \leq 0.001$ (Mann-Whitney non-parametric test). 

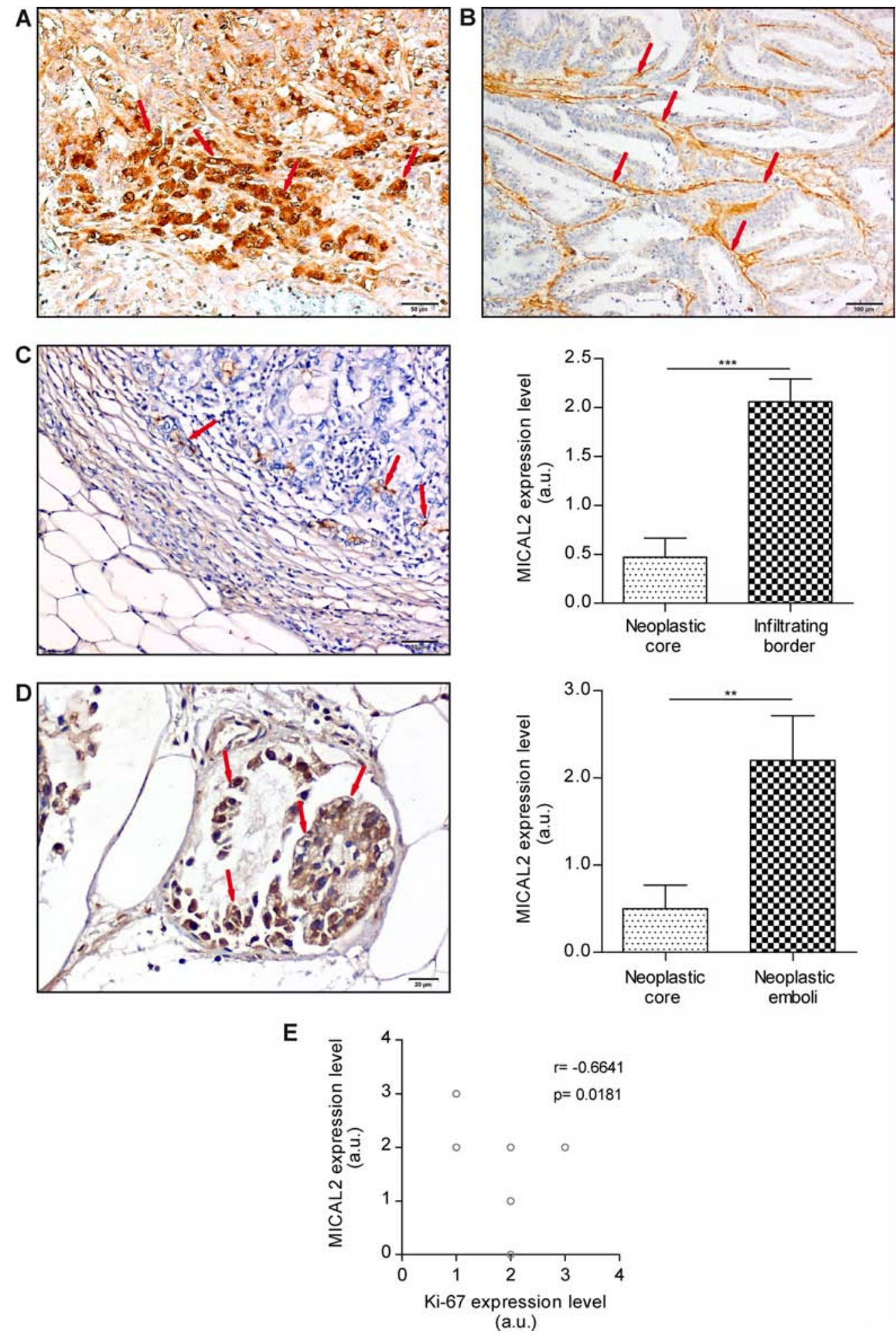

Figure 2: MICAL2 protein is abnormally expressed in primary human GC (IHC analysis). (A) Strong MICAL2-positive immunodecoration of the tumor, mostly as scattered neoplastic cells infiltrating the gastric wall (DAB stain, arrows). Scale bar: $50 \mu \mathrm{m}$. (B) Intestinal-type tumors showed very low to undetectable MICAL2 expression in cancer cells, but strong immunodecoration of neoangiogenic ECs (DAB staining, arrows). Scale bar: $100 \mu \mathrm{m}$. (C) MICAL2-positive cells (arrows) were found at the tumor infiltrating border rather than in the neoplastic core. Scale bar: $50 \mu \mathrm{m}$. MICAL2 mean expression level at the tumor infiltrating edge is higher than in the neoplastic core, $N=17,{ }^{* * *} p \leq 0.001$ (Student's T test for paired samples). (D) MICAL2-positive cells in neoplastic emboli (8/10, 80\%). Scale bar: $20 \mu \mathrm{m}$. MICAL2 expression in emboli was higher than in the neoplastic core, $N=10$, ** $p \leq 0.01$ (Student's $T$ test for paired samples). (E) Qualitative IHC analysis (images not shown) of single immunolabelling indicated Ki-67 expression level inversely correlated with MICAL2 expression. $N=10, r=-0.6641 ; p=0.0181$ (Pearson correlation test). 

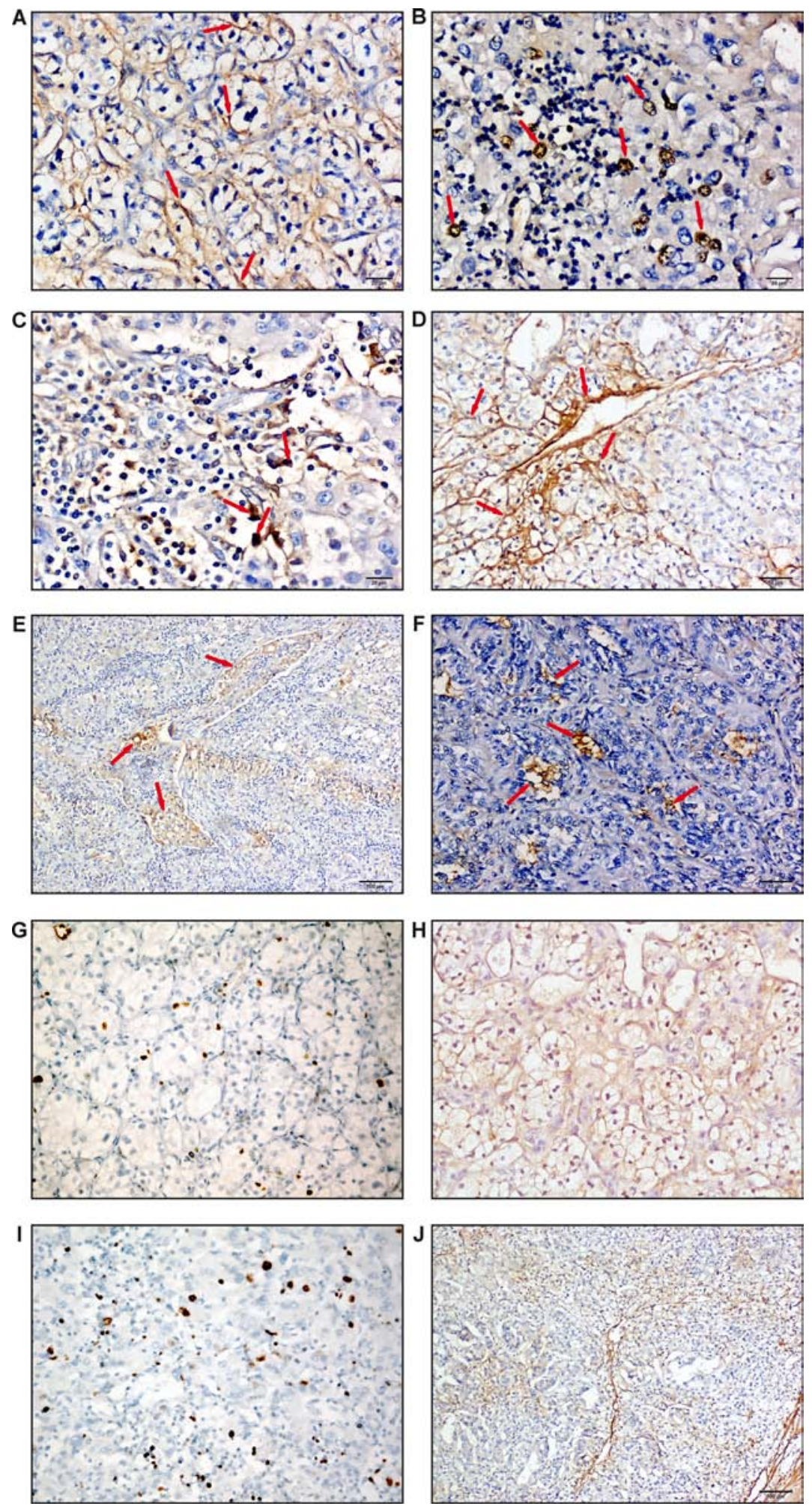

Figure 3: MICAL2 is abnormally expressed in human kidney cancer. IHC analyses (DAB stain, arrows) showed MICAL2 subcellular localization was (A) either cytoplasmic, (B) nuclear, (C) or both, scale bar: $20 \mu \mathrm{m}$. (D) Also in kidney cancer, MICAL2 was highly expressed in neo-angiogenic ECs. Scale bar: $50 \mu \mathrm{m}$. (E), Neoplastic emboli of metastases to the lung showed cells with intense MICAL2-positive immunodecoration (Scale bar: $100 \mu \mathrm{m}$ ), however (F) in the metastatic mass MICAL2 immunolabeling was very low and limited to rare, scattered peripheral cells (Scale bar: $50 \mu \mathrm{m}$ ). Representative images of cancer areas in which: (G) low expression of Ki-67 was associated with $\mathbf{( H )}$ relatively high expression of MICAL2 (sample ID: 10190), and (I) relatively high expression of Ki-67 with (J) low/undetectable expression of MICAL2 in cancer cells, with expression localized to interstitium as evident from low magnification image (sample ID: 14587). 
the elongated, mesenchymal-like shape and scattered distribution on the plate, with minimal if any intercellular contacts, MIC2-KD cells appeared larger, organized in tightly packed clusters, with apico-basal polarity of epithelial cells (Figure 4A).

Through its redox activity, D-MICAL is a direct regulator of F-actin dynamics in neural cells [4]. In non-neural cells, human MICALs are also regulators of actin stress fibers [13]. We stained F-actin to further characterize MIC2-KD phenotype, and observed a clearly different F-actin distribution (Figure 4B). Circumferential actin belts of individual cells, with abundant intracellular puncta, loss of prominent stress fibers, and presence of well-defined rims, indicative of cell-cell junctions in polarized cells of epithelial sheets [19], were visible in MIC2-KD cells, whereas reference cells (CTRL and parental) presented stress fibers and scarse pericellular F-actin, in agreement with [13].

To test the hypothesis that MICAL2 down-regulation leads to MET, we performed immunocytochemistry (ICC) analysis of typical epithelial markers of cell-cell contact, such as Zonula Occludens 1 (ZO-1) at tight junctions, E-cadherin and catenin-beta (CTNNB1) at adherens junctions. These markers were found sharply localized at cell-cell junctions in MIC2-KD cells, designing finely honed cell perimeters, typical of epithelial cells, rather than diffusely distributed in the cytoplasm as in mesenchymal-like reference cells (Figure 4B). Also, MIC2-KD cells showed short and disorganized vimentin filaments, compared with the longer meshes observed in control cells (Figure 4B). Vimentin and CTNNB1 expression was analyzed also by Western blot (WB). In MIC2-KD cells, CTNNB1 level was found strongly decreased (Figure 4C); WB anti-vimentin performed with V9 antibody (an anti-vimentin antibody different from that used for IF) did not yield any signal, as if the corresponding epitope was undetectable.

Signaling pathways activated by intrinsic or extrinsic stimuli responsible for eliciting EMT/MET converge on transcription factors (TFs) ultimately regulating the phenotypic changes, like Snail family zinc finger (SNAI)1 and SNAI2 that impart mesenchymal traits to tumor cells including increased motility and invasiveness, and are responsible for transcriptional repression of E-cadherin [20, 21]. With QRT-PCR the expression of both SNAI1 and SNAI2 resulted undetectable in KD2 and KD14 cells (Figure 4D).

Further, over-expression of MICAL2-tagged cDNA (HA-MICAL2) in flat, cobblestone-like, MICAL2depleted, KD14 cells produced motile, fibroblast-like cells (Figure 4E, 4F), resulting in the rescue of the mesenchymal phenotype of parental 786-O cells. To describe the cell shape change, we performed a quantitative morphometric analysis of cell perimeter, and characterized cell shape with circularity analysis. 786-O reference cells showed a more elongated phenotype (mean circularity values of 0.326 and 0.285 respectively) compared with the two MIC2-
KD cell lines (mean circularity values of 0.651 for KD2 and 0.621 for KD14, Figure 4E). Dividing the population of transfected MIC2-KD14 cells into two groups of lowfluorescence and high-fluorescence cells (HA-MICAL2 ${ }^{\text {low }}$ and HA-MICAL2 ${ }^{\text {high }}$ ) as monitored by IF with anti HAtag antibody, we also noticed a more elongated phenotype with dose-dependency on HA-MICAL2 expression (mean circularity values of 0.244 for HA-MICAL2 $2^{\text {low }}$ and 0.116 for HA-MICAL2 ${ }^{\text {high }}$, Figure 4E).

\section{Knocking-down MICAL2 reduces cell viability, adhesion and motility on 2D surfaces, and invasion properties in 3D matrices of cancer cells in vitro}

To investigate how MIC2-KD affected basic cellular mechanisms like proliferation, cell adhesion, migration and invasion, we performed viability and functional assays in 786-O, MDA-MB-231 and MERO-14 cells. MTT-based cell-growth assays and manual counting showed that reducing MICAL2 mRNA level significantly decreased 786-O and MERO-14 cell viability (Figure 5A, 5B). Since cell adhesion and motility play a key role during EMT, and MICAL2 has been implicated in growth cone dynamics [3, 9], we asked whether MICAL2 expression might affect cancer cell adhesion in vitro. On type-I collagen-coated plates, 786-O KD2 and KD14 cells showed 33\% reduction in adhesion capacity (Figure 5C), and similar results were obtained with MERO-14 KDM1 cells (reduction of 30\%, Figure 5D).

Further, a chemotaxis assay showed KD2 and KD14 cells were not able to cross the blind-well chamber membrane, even in the presence of $10 \%$ FBS (Figure 6A). We also performed a random 2D migration (chemokinesis) assay by plating cells in the presence of a diffuse chemokinetic stimulating agent (10\% FBS). Time-lapse microscopy showed persistent migration by 786-O reference cells when observing single-cell traces obtained during the $15 \mathrm{hr}$ experiments, while KD2 and KD14 cells almost did not leave the origin (Figure 6B and Supplementary Movies). MIC2-KD cells moved randomly (Figure 6C) and more slowly (Figure 6D). We also observed a different behavior of the lamellipodia (Figure 6E, 6F). Reference cells migrated by extending dynamic protrusions with polarized and persistent lamellipodia, well-defined trailing and leading edges. In contrast, most MIC2-KD cells showed dynamic but irregularly shaped lamellipodia, often broader, almost circular, from which multiple, small, narrow and highly dynamic protrusions sticked out (Supplementary Movies and Figure 6E). This quasi radial 'symmetrization' of the cell motility apparatus resembled closely the 'stop signal' caused by prolonged contact [22], although in this case at the presence of very few surrounding cells. These results suggested MICAL2 abatement changed cell membrane protrusion morphology and behavior, and consequently net cell movement in 2D migration. 
A
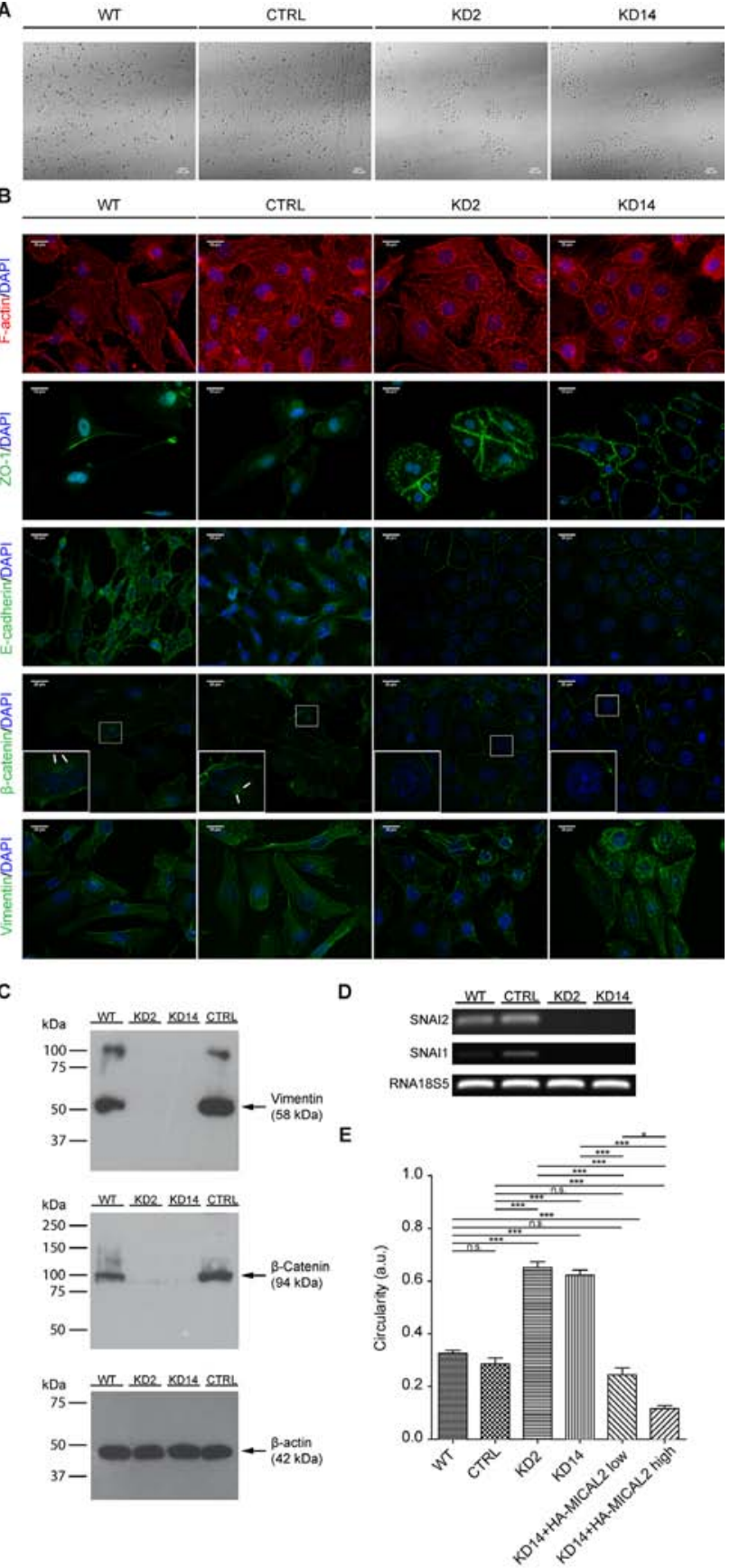

$\mathrm{F}$
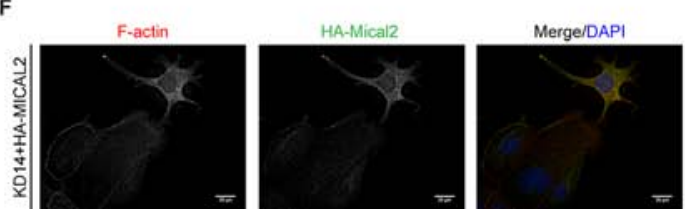

Figure 4: Abating MICAL2 in 786-O kidney cancer cells in vitro induces MET. (A), Morphological analysis in light-transmitted microscopy. Scale bar: $100 \mu \mathrm{m}$. (B), F-actin staining. IF of: E-cadherin, ZO-1, catenin-beta. In KD2 and KD14 cells, these three markers relocated at cell perimeters (as in epithelial cells), rather than being diffusedly cytoplasmic. IF of vimentin with Vim13.2 antibody. In MIC2-KD cells it decorated short and disorganized filaments instead of long meshes observed in control cells. Scale bar of all IF images: $20 \mu \mathrm{m}$. Inset magnification: 3x. (C), Vimentin epitope recognized by V9 antibody was undetectable in KD2 and KD14 cells. (D), Agarose gel run of SNAI1, SNAI2, 18s5 RT-PCR products. (E), KD14 cells transfected with HA-MICAL2 cDNA. The elongated phenotype showed dose-dependency on HA-MICAL2 expression. One-way Anova test and Tukey's Multiple Comparison post-hoc test. In all graph bars, horizontal lines denote mean and SEM. N.s: non significant. ${ }^{*} p \leq 0.05, * * p \leq 0.01,{ }^{* * *} p \leq 0.001$ ). (F), MIC2-KD14 cells expressing HAMICAL2 (shown by anti-HA immunostaining) recovered a mesenchymal-like phenotype with concomitant F-actin redistribution. 
Since several primary carcinoma specimens showed MICAL2-positive cells on the cancer invasive edge and in intravasal tumor emboli (Figure 2C, 2D), we tested the contribution of MICAL2 to invasive properties of cancer cells in vitro. We performed invasion assays with 786-O and MDA-MB-231 cells in 3D collagen matrix to characterize the migratory properties of cancer cells challenged to overcome the stiffness inherent to the surrounding matrix, a condition absent in 2D migration.

In 3D spheroid invasion assay, kidney cancer KD2 and KD14 cells were totally unable to invade the surrounding matrix during the 72 hr-experiment. Breast cancer cells KDM4 also showed reduction of invasion capacity (Figure 7B).

A Viability (786-O cells)
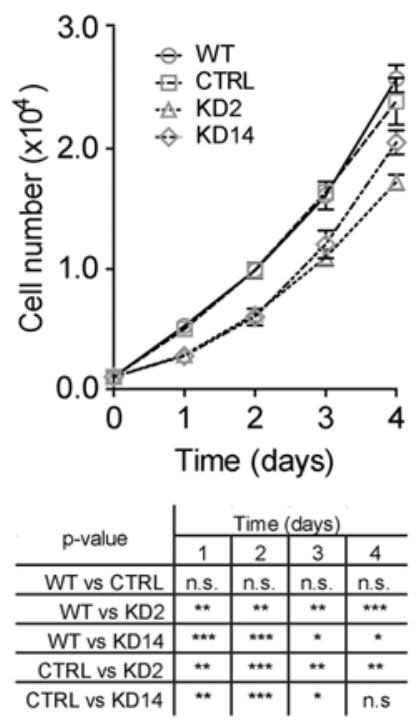

C

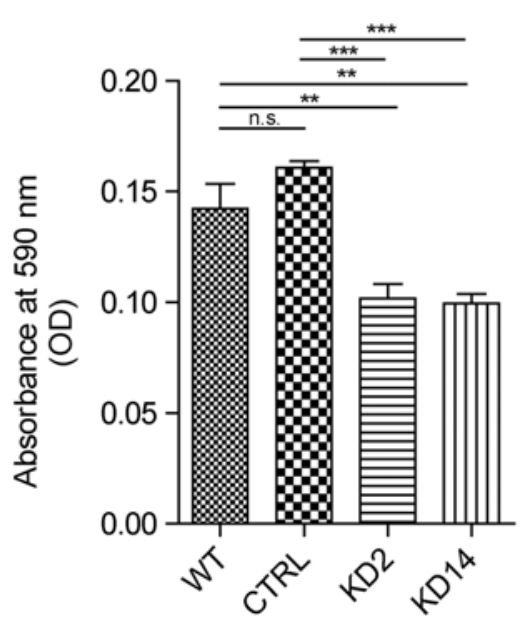

In vertical 3D invasion assay, 786-O MIC2-KD cells run only $33 \%$ of the average distance of cell migration above $20 \mu \mathrm{m}$ performed by reference cells (Figure 7C). Altogether, these results suggested MICAL2 activity is necessary to confer cells the ability to detach from an origin and move within a complex 3D matrix.

\section{MICAL2 is implicated in radical oxygen species (ROS) production in cancer cells}

MICAL proteins display an N-terminal MO domain which binds flavin-adenine-dinucleotide (FAD) and uses the coenzyme nicotinamide-adeninedinucleotide-phosphate (NADPH) in redox reactions to

B Viability (Mero-14 cells)
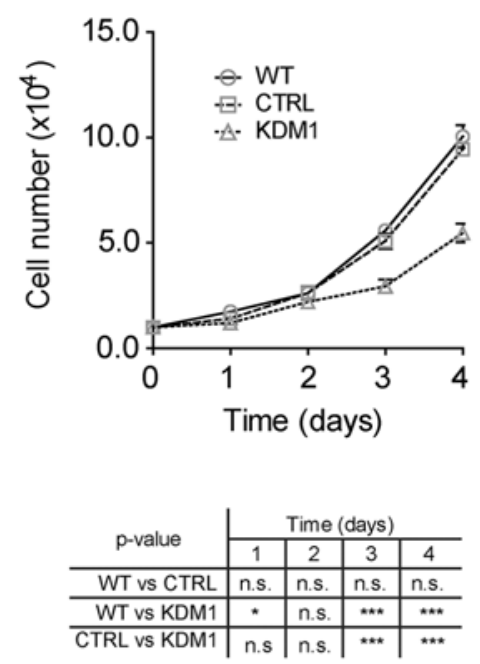

D Adhesion (Mero-14 cells)

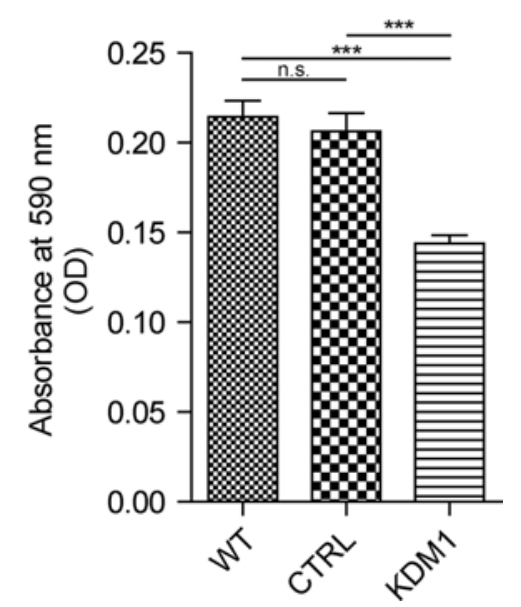

Figure 5: Depleting MICAL2 reduces cancer cells viability and adhesion in vitro. MIC2-KD cells showed significantly decreased viability compared with respective (A) 786-O and (B) MERO-14 WT and CTRL cells. MIC2-KD reduced adhesion of KD2 and KD14 (33\%, (C) and KDM1 (30\%, (D) cells on type-I collagen. One-way Anova test and Tukey’s Multiple Comparison post-hoc test. In all graph bars, horizontal lines denote mean and SEM. N.s.: non significant. ${ }^{*} p \leq 0.05$, ${ }^{* *} p \leq 0.01$, ${ }^{* * *} p \leq 0.001$. 
A

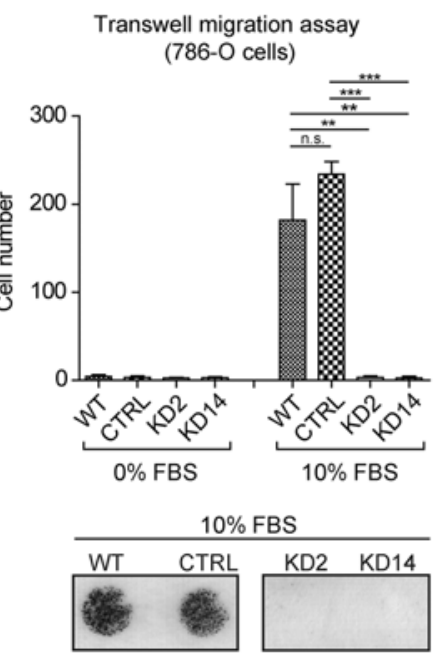

C

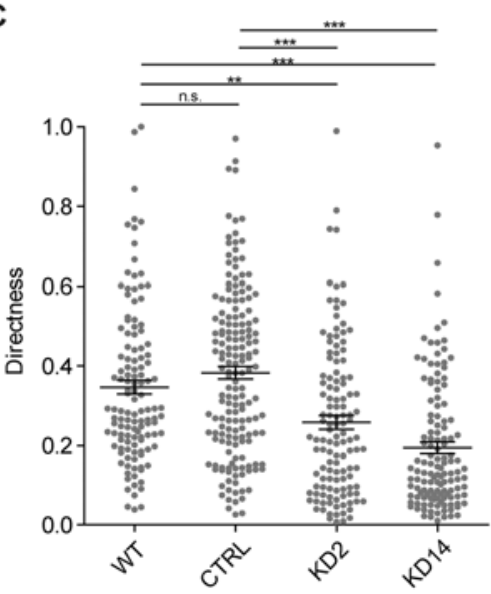

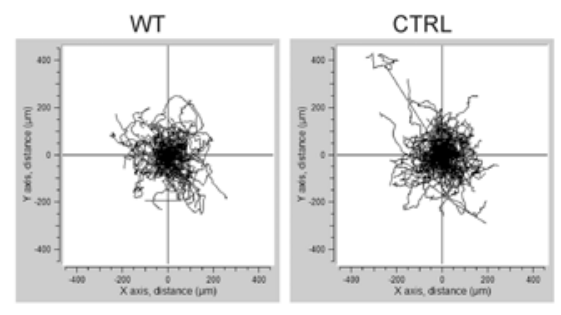

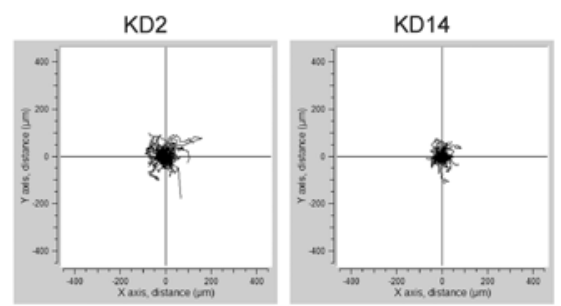

D

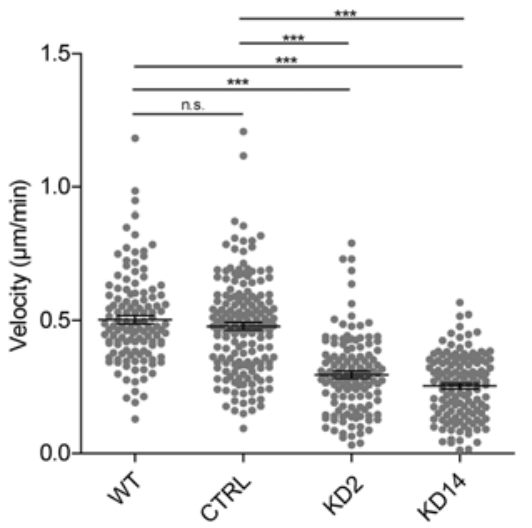

$\mathbf{F}$

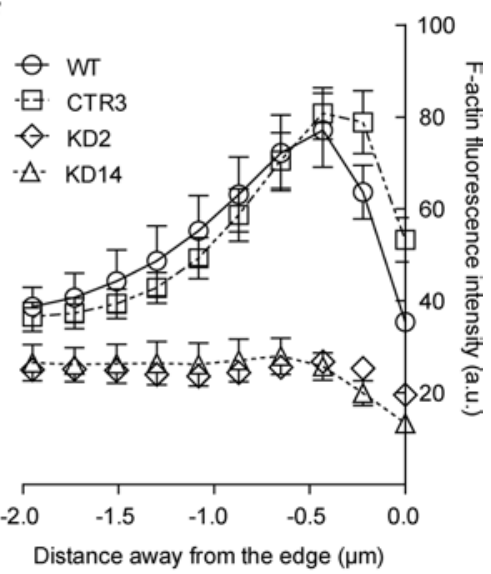

Figure 6: MICAL2 abatement in vitro reduces motility of 786-O ccRCC cells. (A) Transwell migration assay: MIC2-KD cells were not able to cross the $10 \mu \mathrm{m}$-pore membranes of blind-well chambers, even in the presence of $10 \%$ FBS. Lower panel: result of a representative experiment. (B) Single-cell traces of 15-hr chemokinesis assay: KD2 and KD14 cells run an extremely short distance. Directness (C) and Velocity (D), indicators of oriented movement, were reduced. (E) (F)-actin staining with Phalloidin. Scale bar: $20 \mu \mathrm{m}$. Inset magnification: 3.7X. F, Fluorescence intensity derived from Phalloidin staining is measured for $2 \mu \mathrm{m}$ across the cell border; negative distances are meant from the outer side of the contour toward the inner cell side. The peculiar peak of fluorescence intensity, occurring within the first $\mu \mathrm{m}$ away from the leading edge of lamellipodia in reference cells, was lost in KD2 and KD14 cells. A, C and D: One-way Anova test and Tukey's Multiple Comparison post-hoc test. In all graphs, horizontal lines denote mean and SEM. N.s.: non significant. ${ }^{*} p \leq 0.05,{ }^{* *} p \leq 0.01,{ }^{* * *} p \leq 0.001$. 
either directly oxidize substrate proteins and signaling molecules and/or produce ROS [5, 7, 8]. ROS in general and $\mathrm{H}_{2} \mathrm{O}_{2}$ in particular were associated with EMT induction and sustenance [23]. Therefore, we tested the hypothesis that reducing MICAL2 might affect ROS production. 786-O WT, CTRL, KD2 and KD14 cells were treated for 30 minutes with $25 \mathrm{uM}$ 2', 7'-dichlorodihydrofluorescein diacetate ( $\left.\mathrm{H}_{2} \mathrm{DCFDA}\right)$, a cell-permeant ROS probe which is converted in the fluorescent 2', 7'-dichlorodihydrofluorescein $\left(\mathrm{H}_{2} \mathrm{DCF}\right)$ upon oxidation within the cell. Cells were then live imaged with a fluorescent microscope to detect $\mathrm{H}_{2} \mathrm{DCF}$ fluorescence (Figure 8). A three to four folds $\mathrm{H}_{2} \mathrm{DCF}$ fluorescence reduction was found in MIC2-KD clonal lines.

We concluded that MICAL2 activity participates to ROS production, which is in agreement with current literature showing MICAL2-dependent ROS level increase in HeLa cells over-expressing MICAL2 [13].

\section{DISCUSSION}

To search for novel metastatic factors, we investigated a possible role in cancer for MICAL2, a
A

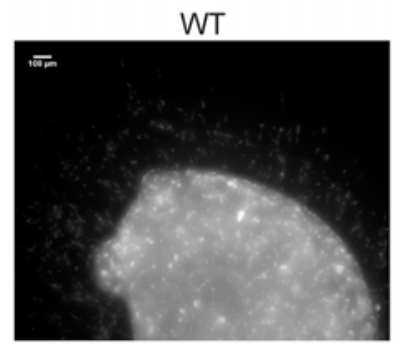

KD2

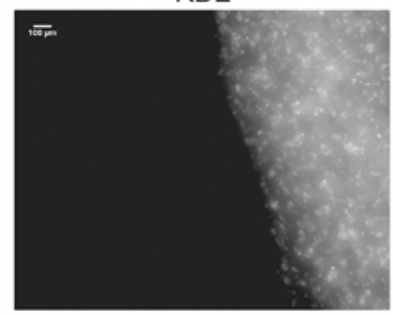

B

3D spheroid invasion assay (MDA-MB-231 cells)

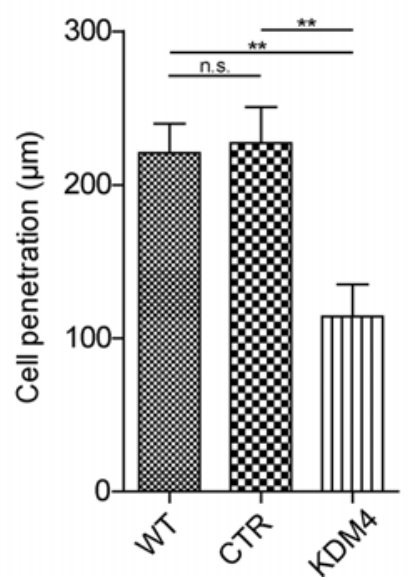

3D spheroid invasion assay

(786-O cells)

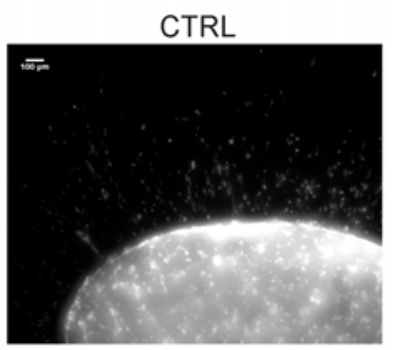

KD14
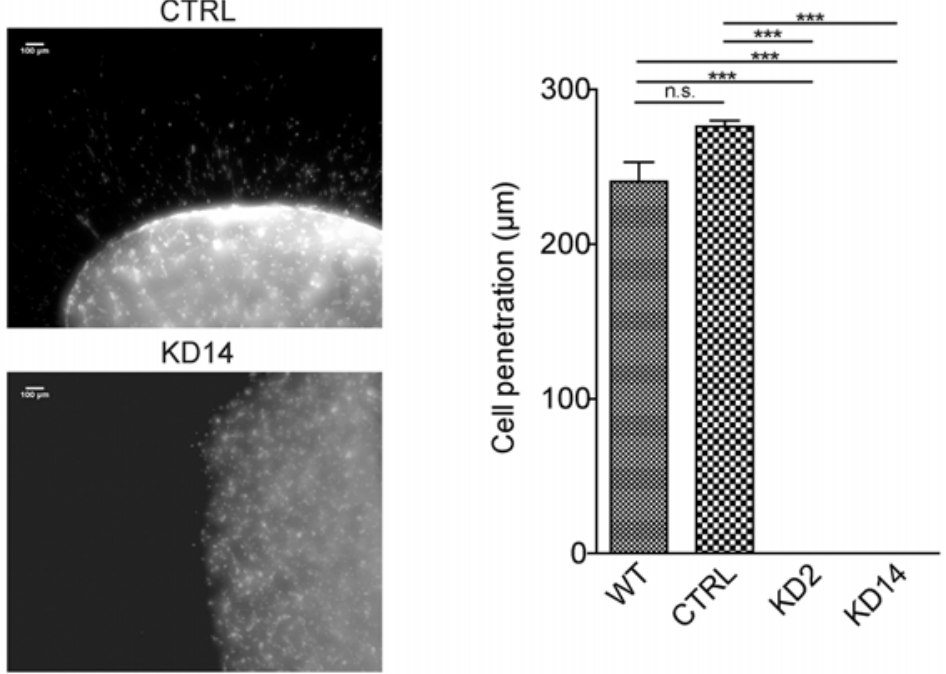

C

Vertical 3D invasion assay

(786-O cells)
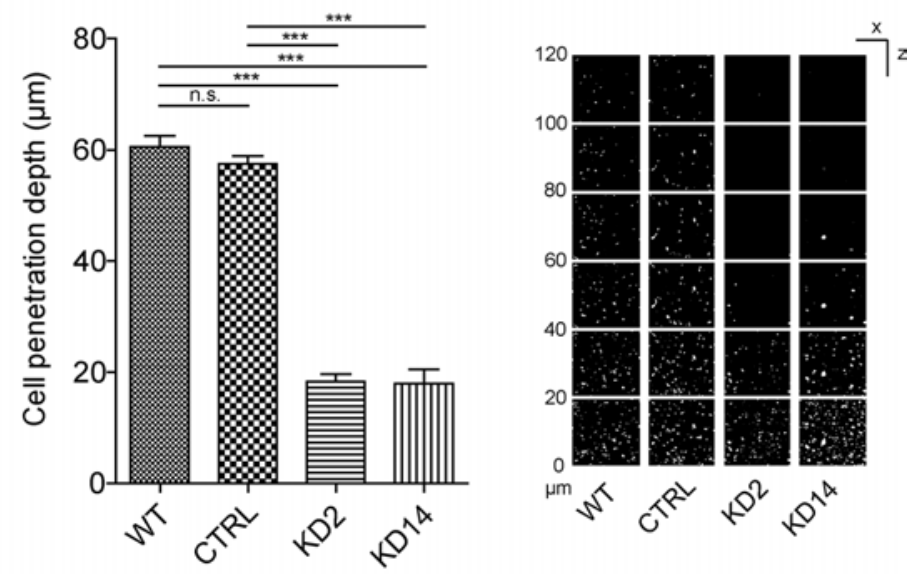

Figure 7: MICAL2 abatement strongly reduces cancer cell 3D invasion properties. (A) Representative result of 3D spheroid invasion assay. (B) KDM4 showed reduced invasion activity. (C) Right: representative result of vertical 3D assays. One-way Anova test and Tukey's Multiple Comparison post-hoc test. In all graphs, horizontal lines denote mean and SEM. N.s.: non significant. * $p \leq 0$, 05 , $* * p \leq 0,01, * * * p \leq 0,001$. 
protein involved in controlling cytoskeleton plasticity, a trait extremely relevant to cell rearrangements and motility in metastatic dissemination.

We found MICAL2 significantly over-expressed in poorly differentiated and undifferentiated gastric and kidney cancer, compared with more differentiated carcinomas. In ccRCC patients, MICAL2 over-expression was significantly associated with metastasis (Figure 1).

All observations in primary and metastatic human tumors suggested that MICAL2 expression is 'on' in a subpopulation of primary cancer cells seemingly detaching from the origin and prone to actively migrate, in individual mode, resist anoikis and travel to distant sites. In brief, are in a state of EMT. In fact, recent data show that MICAL2 is part of a gene signature that specifically characterize highgrade tumors bearing mesenchymal markers [24]. MICAL2 is then turned 'off' following homing at the metastatic site, where MET occurs. The hypothesis of an 'on-off' expression mode during cancer progression, sustained by our in vitro functional analysis, is in line with current literature supporting that cancer spreading depends on mesenchymallike cells located on the cancer invasive front [10, 25].
This matches well with previous studies showing that loss of epithelial markers, like E-cadherin, and gain of mesenchymal markers, like vimentin (both events observed in our in vitro studies) can promote intravasation and early steps of metastasis, but then re-epithelialization is necessary for proliferation of disseminated cells at metastatic sites [26, 27].

Also the inverse correlation with Ki-67 expression level suggested that MICAL2 expression might occur in relatively few (compared with the tumor mass) but critical cells endowed with a motile, invasive phenotype at the tumor edge, rather than proliferating in situ.

So MICAL2 is probably turned on by specific stimuli in the cancer microenvironment and its expression, after having accompanied/sustained cancer cell migration, is turned off at the metastatic site where the intensity of those signals has faded. This allows MET and reprise of proliferation. Consistently, MIC2-KD cells showed in vitro a coherent image of MET revealed by the dramatic reorganization of F-actin and by molecular analysis of indicative markers [28, 29] such as E-cadherin, ZO-1, CTNNB1 and vimentin (Figure 4B), suggesting indirectly

B

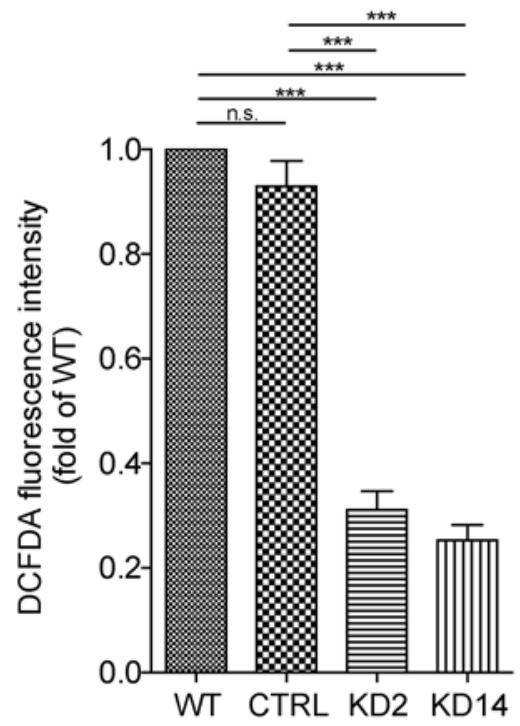

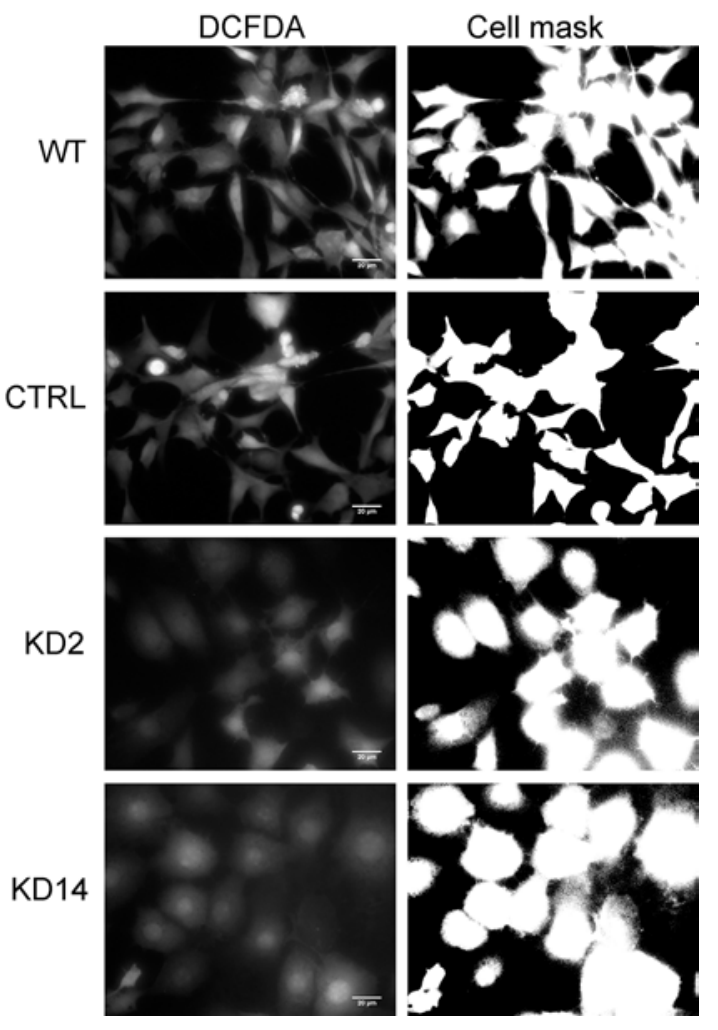

Figure 8: MICAL2 is implicated in radical oxygen species (ROS) production in cancer cells. (A) Detectable ROS levels were present in WT and CTRL cells, by contrast a three to four folds reduction in $\mathrm{H}_{2}$ DCF fluorescence in the MIC2-KD clonal lines was found. (One-way Anova test and Tukey's Multiple Comparison post-hoc test. Horizontal lines denote mean and SEM. Ns: non significant. $\left.{ }^{*} p \leq 0,05,{ }^{* *} p \leq 0,01,{ }^{* * *} p \leq 0,001\right)$. (B) representative micrographs showing $\mathrm{H}_{2} \mathrm{DCF}$ fluorescence in 786-O WT, CTRL, KD2 and KD14 cells (left) and binary mask created in ImageJ by applying a threshold, to visualize cell boundaries (right). 
that MICAL2 regulated function is necessary for formation of proper cell-cell junctions. Interestingly, vimentin and CTNNB1 over-expression and/or cytoplasmic accumulation (as we found in cancer cells expressing high level of MICAL2 in vitro) are both known predictors of hematogenous metastasis in human cancer [30, 31].

MET in MIC2-KD cells occurred through transcriptional reprogramming, indicated by the decrease/ loss of expression of SNAI1 and SNAI2 (Figure 4D), two TFs crucial in initiating/maintaining EMT [19-21]. How this happens was not addressed in this work but, based on information about other MICAL family members [14, 32-36], MICAL2 might take part in vesicular trafficking required for transfer of cell surface receptors or other cell signaling event.

Furthermore, when we reconstituted MICAL2 expression by cDNA transfection in MIC2-KD cells, that have epithelioid phenotype, we obtained a mesenchymal phenotype (Figure 4E, 4F). On one hand this supported the specificity of the results of our knock-down approach and, on the other, more importantly, suggested that the expression/re-expression of MICAL2 is sufficient to induce EMT in epithelial cells.

Overall, all MIC2-KD cell populations generated from cancer cells of different histotypes were found less adherent (Figure 5) and noticeably less capable of migrating (Figure 6) and invading (Figure 7) in 2D and 3D assays in vitro, consistently with a MET phenotype. Directional migration requires cell polarity, but MIC2-KD cells lacked a clearly polarized arrangement in 2D and 3D assays, suggesting that in cancer MICAL2 endogenous over-expression might promote invasion. Often, in metastatic cancers other actin-modifying enzymes were found expressed in deregulated ways. Although that is not a cancer-initiating event it can certainly be a cancerpromoting factor, because expression normalization can revert the metastatic phenotype [38].

Finally, it was proposed that in addition to directly modifying F-actin through redox reactions, MICALs may influence intracellular signaling through generation of ROS as second messengers, in a physiologically restricted manner [9]. ROS have per se notorious pro-metastatic, pro-neoangiogenic effects. Our data showing sharp decrease of ROS production in MIC2-KD cells suggest that endogenous over-expression of MICAL2 in human cancer might generate high ROS level with a consequent cellautonomous, pro-metastatic, pro-angiogenic effect during EMT. MIC2-KD might contribute to ROS decrease and MET also through inhibition of SNAI1, whose expression increases ROS level in cancer cells leading to EMT [39].

In conclusion, this is the first work to show data from human cancer and in vitro analyses suggesting that MICAL2 represents a marker of metastatic disease that promotes migration and invasion of epithelial cancers. MICAL2 might represent a new therapeutic target for thwarting epithelial cancer cell pro-invasive and prometastatic potential.

\section{MATERIALS AND METHODS}

\section{Tissue specimens}

Human paired tumor/normal tissue samples were retrospectively obtained from patients who underwent surgery with curative or palliative intent for primary gastric cancer $(N=64)$ at the Department of Pathological Anatomy of Azienda Ospedaliera Universitaria Senese (Siena, Italy) and the Department of General Surgery and Oncology (University of Siena, Italy), and for lung $(N=27$ NSCLC $)$ and kidney cancer $(N=36$ ccRCC $)$ at the Blokhin Cancer Research Center (Russian Academy of Medical Sciences, Moscow, Russia). The study was done in accordance with the Declaration of Helsinki. Approval was obtained from pertinent ethics committee. All patients gave informed written consent. Patients who had undergone pre-operative radiotherapy or chemotherapy were excluded. Samples were taken in the operating room and stored in liquid nitrogen. In addition to the tumor, a sample of paired normal tissue was collected from the operative specimens at least $10 \mathrm{~cm}$ from the tumor, when applicable. Only samples containing $70-80 \%$ or more tumor cells, and adjacent specimens, fixed in formalin and embedded in paraffin, were used.

Diagnosis and histological grading were assessed using standard criteria by experienced pathologists. Renal cell carcinomas were classified and graded according to [17] and staged according to [40]. Gastric cancers were classified in intestinal, diffuse and mixed histotype according to [16] and staged according to [40].

\section{Cell lines and culturing}

786-O (ATCC CRL-1932), and MDA-MB-231 (ATCC HTB-26) were purchased from ATCC-LGC Standards (Eu), MERO-14 from Sigma.

Cell lines were purchased ad hoc for the study and kept in appropriate growth medium additioned with $100 \mathrm{IU} / \mathrm{ml}$ penicillin, $100 \mu \mathrm{g} / \mathrm{ml}$ streptomycine: $786-\mathrm{O}$ in RPMI-1640 (Sigma-Aldrich) with 10\% heat-inactivated fetal bovine serum (FBS, Gibco), $10 \mathrm{mM}$ HEPES, $1 \mathrm{mM}$ Na pyruvate, $100 \mathrm{mM}$ glutamine; MDA-MB-231 in RPMI-1640 (Sigma-Aldrich) with 10\% FBS (Gibco), $100 \mathrm{mM}$ glutamine; MERO-14 in DMEM (SigmaAldrich) with $15 \%$ FBS (Gibco), $200 \mathrm{mM}$ glutamine. All cell cultures were kept in $5 \% \mathrm{CO}_{2}$ incubator at $37^{\circ} \mathrm{C}$, and routinely tested for mycoplasma contamination (MycoAlert Kit, Lonza).

\section{In silico analysis}

Public bioinformatic resources used for this study: http://www.ncbi.nlm.nih.gov/unigene/, http://www.cbs.dtu. dk/services/NetPhos/, http://web.expasy.org/findmod/, http:// rsbweb.nih.gov/ij/index.html, http://www.genesapiens.org/, http://discover.nci.nih.gov/cellminer/analysis.do. 


\section{RNA and cDNA preparation for retrotranscription-PCR (RT-PCR) and semi-quantitative real time-PCR (QRT-PCR)}

Total RNA was isolated with RNeasy Mini Kit (Qiagen). Purified total RNA samples were quantified spectrophotometrically (NanoDrop ND-1000, NanoDrop Technologies, USA), and quality-checked (Bioanalyzer 2100, Agilent Technologies, USA). cDNA was synthesized using random hexamers and oligo-dT primers (QuantiTect Reverse Transcription Kit, Qiagen).

RT-PCR was performed in 2720 Thermal Cycler (Applied Biosystems, USA), with GoTaq Flexi DNA Polymerase (Promega). QRT-PCR was performed in technical and when applicable biological triplicates, in ABI 7000 PRISM SDS thermal cycler (Applied Biosystems). Data were analyzed through relative quantification with the $-\Delta \Delta \mathrm{Ct}$ method.

Sequence of primers: EEF1A1 (NM_001402.5): 5'-CTTTGGGTCGCTTTGCTGTT-3', 5'-CCGTTCTTC CACCACTGATT-3'; GAPDH (NM_001289746.1); 5'-GC TCATTTCCTGGTATGACAACG-3', 5'-AGGGGTCTAC ATGGCAACTG-3'; beta-actin (NM_001101.3): 5'-AACT GGAACGGTGAAGGTGACAGC-3'，5'-AGAAGTGG GGTGGCTTTTAGG-3'; PPIA (NM_021130.3): 5'-TTC ATCTGCACTGCCAAGAC-3', 5'-TCGAGTTGTCCACA GTCAGC-3'; 18S5 (NR_003286.2): 5'-GTAACCCGT TGAACCCCATT-3', 5'-CCATCCAATCGGTAGTAGC G-3'; MICAL2 (NM_014632.2) : 5'-CAACCCGTGT GTGTCTCATC-3', 5'-GTGGATGCCTGGACAAAGT T-3'; SNAI1 (NM_005985.3): 5'-AATCGGAAGCCTA ACTACAGCG-3', 5'-GTCCCAGATGAGCATTGGCA-3'; SNAI2 (NM_003068.4): 5'-GACCCTGGTTGCTTCA AGGA-3', 5'-TGTTGCAGTGAGGGCAAGAA-3'.

Primers were used at the following conditions: 2 min at $95^{\circ} \mathrm{C}$; 33 cycles of $30 \mathrm{sec}$ at $95^{\circ} \mathrm{C}, 30 \mathrm{sec}$ at $58^{\circ} \mathrm{C}$, $15 \mathrm{sec}$ at $72^{\circ} \mathrm{C} ; 5 \mathrm{~min}$ at $72^{\circ} \mathrm{C}$. Amplicons were sequenceverified (3730 DNA Analyzer automated sequencer, Applied Biosystems).

For QRT-PCR on human cancer specimens, Hs01121791_m1 (human MICAL2) and Hs01111406_g1 (human RPN1) TaqMan GeneSpecific Assays FAM dyelabeled (Applied Biosystems) and TaqMan Universal PCR Master Mix (Applied Biosystems) were used. Human RPN1 was used as reference gene. RPN1 mRNA level variability was not higher than 2-fold in tumor $(\mathrm{T})$ and normal $(\mathrm{N})$ tissues. The reference gene for QRT-PCR on cancer cell lines was $18 \mathrm{~S} 5$.

\section{Western blotting, immunofluorescence, immunohistochemistry}

Western blotting (WB) was performed according to standard protocols. Immunofluorescence (IF) analysis was performed as in [41]. Immunohistochemistry (IHC) was performed as in [42]. ICC with DAB staining was performed as in [43].

Cells fixed for IF analysis were incubated with primary and secondary antibody solutions, DAPI (SigmaAldrich), and mounted in Aqua PolyMount (Polysciences, USA).

IHC was performed as in [42] with avidin-biotin complex technique on paraffin embedded sections rehydrated and treated with $\mathrm{H}_{2} \mathrm{O}_{2}$ before incubation with primary and secondary antibody solutions, and pertinent substrate (CN/DAB Substrate Kit, Thermo Scientific), and finally mounted in Canada Balm (Bioptika).

Primary antibodies for WB: rabbit polyclonal anti-MICAL2 (1:1000, generated and validated in our laboratory as in [44], see Supplementary Figure 1), antiCTNNB1 (1:1000, Thermo Scientific, USA), anti-vimentin V9 (1:5000, Sigma), anti-beta-actin (1:7000, SigmaAldrich). Secondary antibodies: HRP-conjugated goat antirabbit (Pierce); HRP-conjugated goat anti-mouse (Pierce).

Primary antibodies for IF: rabbit polyclonal anti-MICAL2 (1:1000, generated and validated in our laboratory as in [44], see Supplementary Figure 1), anti-Ecaderin PA5-27187 (1:100 Thermo Scientific, USA), antiZO-1 40-2300 N-term (1:500, Invitrogen, USA), anti-HA (1:200, Sigma), anti-CTNNB1 (1:100, Thermo Scientific, USA), anti-vimentin VIM 13.2 (1:500, Sigma). Secondary antibodies: Goat anti-rabbit Alexa Fluor 488 (and 568, not shown): 1:1000 (Molecular Probes, USA). Rabbit antimouse Alexa Fluor 568: 1:1000 (Molecular Probes, USA).

Primary antibodies for IHC: rabbit polyclonal antiMICAL2 (1:2500 or 1:1500, generated in our laboratory as in [44], see Supplementary Figure 1), anti Ki-67 (Novocastra Reagents, Leica Biosystems, Australia), antiMICAL1 (1:100, Santa Cruz, USA), anti-MICAL3 (1:100, Santa Cruz, USA). Secondary antibodies provided by VECTASTAIN Elite ABC Kit (Vector Laboratories, USA).

C.V. and A.P. evaluated the results. The immunoreaction was evaluated in arbitrary units (a.u.) in a range from 0 a.u. to 4 a.u. on the bases of antigenpositive cell percentage in the region of interest $(0 \%=0$ a.u.; $1 \%-25 \%=1$ a.u.; $26 \%-50 \%=2$ a.u.; $51 \%-75 \%=3$ a.u.; $76 \%-100 \%=4$ a.u.).

\section{Staining of F-actin}

F-actin was stained with 488 Acti-stain and 568 Phalloidin (Cytoskeleton).

\section{Cell circularity}

After tracing manually the cell perimeters, cell circularity was measured using the circularity plugin built in ImageJ, according to the equation: Circularity $C=4 \pi A / P^{2}$ ( $P=$ cell perimeter, $A=$ cell area). 


\section{Cell viability assay}

Cell proliferation and viability were tested with manual counting and/or with colorimetric assay based on 3-(4, 5-Dimethylthiazol-2-yl)-2, 5-diphenyltetrazolium bromide (MTT, Sigma-Aldrich), starting from $10^{3}$ cells, for 4 days. OD values at $590 \mathrm{~nm}$ (ETI-SYSTEM Fast Reader, Sorin Biomedica, Italy) were plotted against a cell line-specific reference curve to infer the corresponding cell number.

\section{D cell adhesion and motility assays}

Adhesion was evaluated according to standard protocols by plating $10^{4}$ cells on vessels coated with $0.1 \mu \mathrm{g} / \mu \mathrm{l}$ type-I collagen (Sigma-Aldrich). Cells were challenged after $1 \mathrm{hr}$ (786-O) or 15 minutes (MERO-14) from plating, then colored with crystal-violet solution and read at $590 \mathrm{~nm}$ (ETI System Fast Reader, Sorin Biomedica, Italy).

Chemotaxis assay was performed with a modified Boyden Chamber mounting $10 \mu \mathrm{m}$ polycarbonate membrane (NeuroProbe, USA). $8 \times 10^{3}$ cells were seeded in the upper chamber with serum-free growth medium and incubated for $16 \mathrm{hrs}$. The lower chamber contained growth medium plus $10 \%$ FBS (or no FBS for negative control). Staining was performed with Diff-Quick (Medion Diagnostics, Switzerland).

For chemokinesis assay, $2 \times 10^{3}$ cells were seeded in cell growth medium with $10 \%$ FBS. Live imaging was performed (15 hrs) with a microscope-fitting a custommade $5 \% \mathrm{CO}_{2}$ incubation chamber. Directness represents a measure of the cell's tendency to travel in a straight line. It is calculated as the ratio of the Euclidian distance and the accumulated distance in time: $D=d_{\mathrm{E}} / d_{\mathrm{A}}$. $D=1$ indicates a straight-line migration between start and end points [45].

Velocity is the ratio between the accumulated distance and time (experiment duration): $V=d_{\mathrm{A}} / T$, where $T=$ total experiment time.

\section{D invasion assays}

3D spheroid invasion assay and 3D vertical assay were performed as in [46] starting with $5 \times 10^{4}$ cells embedded in droplets of RPMI-1640 (Sigma-Aldrich) and $2.2 \mathrm{mg} / \mathrm{ml}$ type-I collagen (BD Biosciences), at $\mathrm{pH}$ 7.4. Z-stack images were captured every $50 \mu \mathrm{m}$.

$3 \mathrm{D}$ vertical assay [38] was performed starting with $2 \times 10^{5}$ cells/ml resuspended in RPMI-1640 with $2.2 \mathrm{mg} /$ $\mathrm{ml}$ type-I collagen, at $\mathrm{pH} 7.4$, and seeded in $50 \mu$-aliquots in wells pre-coated with $3 \%$ heat-inactivated BSA. Cells were stained with YoPro and photographed along Z-axes through high-density collagen-I. Z-stack images were captured each $5 \mu \mathrm{m}$ from the well bottom, up to $120 \mu \mathrm{m}$ upward, toward the chemoattractant.

\section{Image acquisition and processing}

Images for analysis of IF and F-actin staining were acquired in z-stack with NIS-Elements software (Nikon) and deconvoluted with the associated deconvolution software package, using a fluorescence microscope Nikon Eclipse Ti (Nikon) equipped with 40x DIC M Plan Fluor objective (Nikon) and DS-Qi1Mc-U2 12 bit (Nikon) camera.

Images of ICC on agar-embedded cells and of IHC: Olympus BX43 equipped with camera Olympus DV20 and Cell Sens Dimension software.

Chemotaxis images: Zeiss Axioskop 40 microscope (Zeiss) equipped with $5 \mathrm{x}$ objective (Zeiss), through AxioCam MRc 12 bit camera (Zeiss) and AxioVision software (Zeiss).

Chemokinesis live imaging: Nikon Eclipse Ti microscope (Nikon) and ORCA camera (Hamamatsu) equipped with 20x Ph2 DM Plan Apo objective (Nikon) and NIS-Elements software (Nikon). Images were analyzed with Manual Tracking plugin of ImageJ suite (NIH, USA), following each individual cell to the end of the experiment or to mitosis. Data were analyzed with Chemotaxis and Migration Tool 2.0 (Ibidi).

3D matrix invasion assays: acquired with NISElements software (Nikon) and deconvoluted with the associated deconvolution software, using a Nikon Eclipse Ti microscope (Nikon). It was equipped with $4 \mathrm{x}$ Achromat objective (Nikon) and DS-Qi1Mc-U2 12 bit camera (Nikon) in case of spheroid assay; it was equipped with 20x Ph2 DM Plan Apo objective (Nikon) and ORCA camera (Hamamatsu) in case of vertical assay. Image analysis was performed with ImageJ software (NIH, USA).

\section{Statistical analysis}

Data of protein IHC are indicated as mean \pm SEM and analyzed with Student's $t$ test for paired samples.

Data from gene expression analysis in cell lines and cell circularity, viability, adhesion, chemotaxis, chemokinesis and 3D matrix invasion assays were obtained from more than three independent experiments (each performed with triplicates). They are expressed as mean \pm SEM and analyzed with one-way Anova test and Tukey's Multiple Comparison post-hoc test.

Gene expression analysis in patients' samples ( $N$ versus $T$ ) was evaluated with the non-parametric MannWhitney test.

Protein expression correlation was analyzed with Pearson Correlation test, $r=-0.6641$.

Always, difference between means was judged statistically significant for $p \leq 0.05$.

All statistical procedures were performed using GraphPad software. 


\section{ACKNOWLEDGMENTS}

pCMV6-HA-MICAL2 was a kind gift of Steve Caplan (University of Nebraska, USA). Immunogenic peptides were a kind gift of Renato Longhi (Istituto di Chimica delle Macromolecole, National Research Council, Milan, Italy).

The authors thank Silvia Burchielli (Center for Experimental Biomedicine, Fondazione Monasterio, Pisa, Italy) and Mr. Stefano Mazzoni (University of Pisa, Department of Experimental Pathology, Pisa, Italy) for technical help with rabbits immunization procedures; Maria Teresa Del Vecchio (Department of Human Pathology and Oncology, University of Siena, Siena, Italy) for help with histological analysis of human kidney cancer samples; Valeria Pascale for help with preparation of a set of RNA samples; Gualtiero Pelosi for help with histology image acquisition, and Michael I. Lerman and Antonio Musio for critical reading of the manuscript.

\section{GRANT SUPPORT}

This work was supported by a grant from the Istituto Toscano Tumori, Regione Toscana, and from a grant for cooperation between the Italian National Research Council and the Russian Academy of Sciences to D.A., fellowships from the Doctoral School in Life Sciences of Scuola Superiore Sant'Anna to S.M. and I.B., from the BIOS Doctoral School of the University of Pisa to R.L., from Regione Toscana co-funded by Kayser Italia srl to M.B., and from RAS Presidium Program "Molecular and Cellular Biology" to A.D. Part of this work was performed at EIMB RAS “Genome” Center (http://www.eimb.ru/ RUSSIAN_NEW/INSTITUTE/ccu_genome_c.php) under the financial support by the Ministry of Education and Science of the Russian Federation (Contract 14.621.21.0001, project RFMEFI62114X0001).

\section{CONFLICTS OF INTEREST}

The authors disclose no potential conflicts of interest.

\section{REFERENCES}

1. Pollard TD, Borisy GG. Cellular motility driven by assembly and disassembly of actin filaments. Cell. 2003; 112:453-65.

2. Blanchoin L, Boujemaa-Paterski R, Sykes C, Plastino J. Actin dynamics, architecture, and mechanics in cell motility. Physiol Rev. 2014; 94:235-63.

3. Terman JR, Mao T, Pasterkamp RJ, Yu H-H, Kolodkin AL. MICALs, a family of conserved flavoprotein oxidoreductases, function in plexin-mediated axonal repulsion. Cell. 2002; 109:887-900.
4. Suzuki T, Nakamoto T, Ogawa S, Seo S, Matsumura T, Tachibana K, Morimoto C, Hirai H. MICAL, a novel CasL interacting molecule, associates with vimentin. J Biol Chem. 2002; 277:14933-41.

5. Hung R-J, Pak CW, Terman JR. Direct redox regulation of F-actin assembly and disassembly by Mical. Science. 2011; 334:1710-3.

6. Hung RJ, Yazdani U, Yoon J, Wu H, Yang T, Gupta N, Huang Z, van Berkel WJ, Terman JR. Mical links semaphorins to F-actin disassembly. Nature. 2010; 463: 823-7.

7. Nadella M, Bianchet MA, Gabelli SB, Barrila J, Amzel LM. Structure and activity of the axon guidance protein MICAL. Proc Natl Acad Sci U S A. 2005; 102:16830-5.

8. Siebold C, Berrow N, Walter TS, Harlos K, Owens RJ, Stuart DI, Terman JR, Kolodkin AL, Pasterkamp RJ, Jones EY. High-resolution structure of the catalytic region of MICAL (molecule interacting with CasL), a multidomain flavoenzyme-signaling molecule. Proc Natl Acad Sci U S A. 2005; 102:16836-41.

9. Pasterkamp RJ, Dai HN, Terman JR, Wahlin KJ, Kim B, Bregman BS, Popovich PG, Kolodkin AL. MICAL flavoprotein monooxygenases: expression during neural development and following spinal cord injuries in the rat. Mol Cell Neurosci. 2006; 31:52-69.

10. Thiery JP, Acloque H, Huang RY, Nieto MA. Epithelialmesenchymal transitions in development and disease. Cell. 2009; 139:871-90.

11. Ashida S, Furihata M, Katagiri T, Tamura K, Anazawa Y, Yoshioka H, Miki T, Fujioka T, Shuin T, Nakamura Y, Nakagawa H. Expression of novel molecules, MICAL2PV (MICAL2 prostate cancer variants), increases with high Gleason score and prostate cancer progression. Clin Cancer Res. 2006; 12:2767-73.

12. Zhu LY, Zhang WM, Yang XM, Cui L, Li J, Zhang YL, Wang YH, Ao JP, Ma MZ, Lu H, Ren Y, Xu SH, Yang GD, et al. Silencing of MICAL-L2 suppresses malignancy of ovarian cancer by inducing mesenchymalepithelial transition. Cancer Lett. 2015; doi: 10.1016/j. canlet.2015.04.002. [Epub ahead of print].

13. Giridharan SSP, Rohn JL, Naslavsky N, Caplan S. Differential regulation of actin microfilaments by human MICAL proteins. J Cell Sci. 2012; 125: 614-24.

14. Grigoriev I, Yu KL, Martinez-Sanchez E, Serra-Marques A, Smal I, Meijering E, Demmers J, Peränen J, Pasterkamp RJ, van der Sluijs P, Hoogenraad CC, Akhmanova A. Rab6, Rab8, and MICAL3 cooperate in controlling docking and fusion of exocytotic carriers. Curr Biol. 2011; 21: 967-74.

15. Schmidt EF, Shim S-O, Strittmatter SM. Release of MICAL autoinhibition by semaphorin-plexin signaling promotes interaction with collapsin response mediator protein. J Neurosci. 2008; 28:2287-97. 
16. Lauren P. The two histological main types of gastric carcinoma: diffuse and so-called intestinal-type carcinoma. an attempt at a histo-clinical classification. Acta Pathol Microbiol Scand. 1965; 64:31-49.

17. Pathology and Genetics of Tumours of the Urinary System and Male Genital Organs. Ebele JN, Sauter G, Epstein JI and Sesterhenn IA, editors. World Health Organization Classification of Tumours 2004.

18. Gerdes J, Lemke H, Baisch H, Wacker HH, Schwab U, Stein H. Cell cycle analysis of a cell proliferation-associated human nuclear antigen defined by the monoclonal antibody Ki-67. J Immunol. 1984; 133:1710-5.

19. Farquhar MG, Palade GE. Junctional complexes in various epithelia. J. Cell Biol. 1963; 17:375-412.

20. Nieto MA. The snail superfamily of zinc-finger transcription factors. Nat Rev Mol Cell Biol. 2002; 3:155-66.

21. Barrallo-Gimeno A, Nieto MA. The Snail genes as inducers of cell movement and survival: implications in development and cancer. Development. 2005; 132: 3151-3161.

22. Dustin ML. Hunter to gatherer and back: immunological synapses and kinapses as variations on the theme of amoeboid locomotion. Annu Rev Cell Dev Biol. 2008; 24: 577-96.

23. Giannoni E, Parri M, Chiarugi P. EMT and oxidative stress: a bidirectional interplay affecting tumor malignancy. Antioxid Redox Signal. 2012; 16:1248-63.

24. Shaul YD, Freinkman E, Comb WC, Cantor JR, Tam WL, Thiru P, Kim D, Kanarek N, Pacold ME, Chen WW, Bierie B, Possemato R, Reinhardt F, et al. Dihydropyrimidine accumulation is required for the epithelial-mesenchymal transition. Cell. 2014; 158:1094-109.

25. Fidler IJ, Poste G. The "seed and soil" hypothesis revisited. Lancet Oncol. 2008; 9:808.

26. Ocaña OH1, Córcoles R, Fabra A, Moreno-Bueno G, Acloque H, Vega S, Barrallo-Gimeno A, Cano A, Nieto MA. Metastatic colonization requires the repression of the epithelial-mesenchymal transition inducer Prrx1. Cancer Cell. 2012; 22:709-24.

27. Tsai JH1, Donaher JL, Murphy DA, Chau S, Yang J. Spatiotemporal regulation of epithelial-mesenchymal transition is essential for squamous cell carcinoma metastasis. Cancer Cell. 2012; 22:725-36.

28. Shapiro L, Weis WI. Structure and biochemistry of cadherins and catenins. Cold Spring Harb Perspect Biol. 2009; 1:a003053.

29. Lee JM, Dedhar S, Kalluri R, Thompson EW. The epithelialmesenchymal transition: new insights in signaling, development, and disease. J. Cell Biol. 2006; 172:973-981.

30. Li M, Zhang B, Sun B, Wang X, Ban X, Sun T, Liu Z, Zhao X. A novel function for vimentin: the potential biomarker for predicting melanoma hematogenous metastasis. Exp Clin Cancer Res. 2010; 29:109.
31. Maruyama K, Ochiai A, Akimoto S, Nakamura S, Baba S, Moriya Y, Hirohashi S. Cytoplasmic beta-catenin accumulation as a predictor of hematogenous metastasis in human colorectal cancer. Oncology. 2000; 59:302-9.

32. Bachmann-Gagescu R, Dona M, Hetterschijt L, Tonnaer E, Peters T, de Vrieze E, Mans DA, van Beersum SE, Phelps IG, Arts HH, Keunen JE, Ueffing M, Roepman R, et al. The Ciliopathy Protein CC2D2A Associates with NINL and Functions in RAB8-MICAL3-Regulated Vesicle Trafficking. PLoS Genet. 2015; 11:e1005575.

33. Reinecke JB, Katafiasz D, Naslavsky N, Caplan S. Regulation of Src trafficking and activation by the endocytic regulatory proteins MICAL-L1 and EHD1. J Cell Sci. 2014; 127:1684-98.

34. Rahajeng J, Giridharan SS, Cai B, Naslavsky N, Caplan S. MICAL-L1 is a tubular endosomal membrane hub that connects Rab35 and Arf6 with Rab8a. Traffic. 2012; 13:82-93.

35. Abou-Zeid N, Pandjaitan R, Sengmanivong L, David V, Le Pavec G, Salamero J, Zahraoui A. MICAL-like1 mediates epidermal growth factor receptor endocytosis. Mol Biol Cell. 2011; 22:3431-41.

36. Sharma M, Giridharan SS, Rahajeng J, Naslavsky N, Caplan S. MICAL-L1 links EHD1 to tubular recycling endosomes and regulates receptor recycling. Mol Biol Cell. 2009; 20: 5181-94.

37. Lundquist MR, Storaska AJ, Liu TC, Larsen SD, Evans T, Neubig RR, Jaffrey SR. Redox modification of nuclear actin by MICAL-2 regulates SRF signaling. Cell. 2014; 156:563-76.

38. Lambrechts A, Van Troys M, Ampe C. The actin cytoskeleton in normal and pathological cell motility. Int $\mathrm{J}$ Biochem Cell Biol. 2004; 36:1890-909. Review.

39. Barnett P1, Arnold RS, Mezencev R, Chung LW, Zayzafoon M, Odero-Marah V. Snail-mediated regulation of reactive oxygen species in ARCaP human prostate cancer cells. Biochem Biophys Res Commun. 2011; 404:34-9.

40. System of Clinic-Morphological Classification of Tumors (TNM, VII ed. 2010).

41. Angeloni D, Danilkovitch-Miagkova A, Ivanova T, Braga E, Zabarovsky E, Lerman MI. Hypermethylation of Ron proximal promoter associates with lack of full-length Ron and transcription of oncogenic short-Ron from an internal promoter. Oncogene. 2007; 26:4499-512.

42. Rocchiccioli S, Pelosi G, Rosini S, Marconi M, Viglione F, Citti L, Ferrari M, Trivella MG, Cecchettini A. Secreted proteins from carotid endarterectomy: an untargeted approach to disclose molecular clues of plaque progression. J Transl Med. 2013; 11:260.

43. Rhodes A, Jasani B, Anderson E, Dodson AR, Balaton AJ. Evaluation of HER-2/neu immunohistochemical assay sensitivity and scoring on formalin-fixed and paraffinprocessed cell lines and breast tumors: a comparative study 
involving results from laboratories in 21 countries. Am J Clin Pathol. 2002; 118:408-17.

44. Angeloni D, Longhi R, Fraschini F. Production and characterization of antibodies directed against the human melatonin receptors Mel-1a (mt1) and Mel-1b (MT2). Eur J Histochem. 2000; 44:199-204.

45. Semmling V, Lukacs-Kornek V, Thaiss CA, Quast T, Hochheiser K, Panzer U, Rossjohn J, Perlmutter P, Cao J, Godfrey DI, Savage PB, Knolle PA, Kolanus W, et al.
Alternative cross-priming through CCL17-CCR4-mediated attraction of CTLs toward NKT cell-licensed DCs. Nat Immunol. 2010; 11:313-20.

46. Friedl P, Locker J, Sahai E, Segall JE. Classifying collective cancer cell invasion. Nat Cell Biol. 2012; 14:777-83. 